\title{
BMJ Open Play \& Grow: prospective observational cohort of toddlers to inform obesity prevention, Columbus, Ohio, USA
}

\author{
Andria Parrott, ${ }^{1}$ Bharathi J Zvara, ${ }^{2}$ Sarah A Keim, ${ }^{1,3}$ Rebecca Andridge, ${ }^{4}$ \\ Sarah E Anderson (i) ${ }^{3}$
}

To cite: Parrott A, Zvara BJ, Keim SA, et al. Play \& Grow: prospective observational cohort of toddlers to inform obesity prevention, Columbus, Ohio, USA. BMJ Open 2022;12:e055490. doi:10.1136/ bmjopen-2021-055490

- Prepublication history for this paper is available online. To view these files, please visit the journal online (http://dx.doi org/10.1136/bmjopen-2021055490).

Received 14 July 2021 Accepted 27 October 2021

Check for updates

(c) Author(s) (or their employer(s)) 2021. Re-use permitted under CC BY-NC. No commercial re-use. See rights and permissions. Published by BMJ.

${ }^{1}$ Center for Biobehavioral Health, Abigail Wexner Research Institute at Nationwide Children's Hospital, Columbus, Ohio, USA

${ }^{2}$ Gillings School of Public Health, The University of North Carolina at Chapel Hill, Chapel Hill, North Carolina, USA

${ }^{3}$ Division of Epidemiology, The Ohio State University College of Public Health, Columbus, Ohio, USA

${ }^{4}$ Division of Biostatistics, The Ohio State University College of Public Health, Columbus, Ohio, USA

Correspondence to

Dr Sarah E Anderson; anderson.1767@osu.edu

\section{ABSTRACT}

Purpose Obesity prevention is increasingly focused on early childhood, but toddlers have not been well-studied, and children born preterm are frequently excluded. The Play \& Grow Cohort was established to investigate child growth in relation to parent-child interactions in mealtime and non-mealtime settings.

Participants Between December 2017 and May 2019, 300 toddlers and primary caregivers were recruited from records of a large paediatric care provider in Columbus, Ohio, USA. This report describes recruitment of the cohort and outlines the data collection protocols for two toddler and two preschool-age visits. The first study visit coincided with enrolment and occurred when children ( $57 \%$ boys) were a mean (SD) calendar age of $18.2(0.7)$ months. Findings to date Children in the cohort are diverse relative to gestational age at birth $(16 \%, 28-31$ completed weeks' gestation; 21\%, 32-36 weeks' gestation; 63\%, $\geq 37$ weeks' gestation) and race/ethnicity (8\%, Hispanic; $35 \%$, non-Hispanic black; $46 \%$, non-Hispanic white). Caregivers enrolled in the cohort are primarily the child's biological mother $(93 \%)$ and are diverse in age (range 18-54 years), education (23\%, high school or less; $20 \%$ graduate degree) and annual household income (27\%, $<$ US\$20 000 24\%, >US\$90 000). Parent-child interactions were video-recorded during play in the laboratory at 18 months $(\mathrm{n}=299)$ and during play, reading and mealtime in the home $(n=284)$ at 24 months. The preschool phase of the study was impacted by COVID-19. Parentchild interactions were video-recorded during play and mealtime at home at 36 months $(n=141)$ and during a standardised buffet meal in the laboratory at 42 months $(n=50)$. Caregivers unable to participate in face-to-face visits due to COVID-19 completed questionnaires.

Future plans Assessment during middle childhood is being planned. Future visits will include anthropometric measurements and parent-child interactions at mealtime. School-based outcomes are additionally being considered.

\section{INTRODUCTION}

When children are young, families establish routines, set expectations and develop patterns of interaction that shape future health behaviours and the home environment. ${ }^{1}$ How parents feed children (food parenting practices) can influence children's food consumption and preferences, and are

\section{Strengths and limitations of this study}

- Observational cohort of 300 diverse families enrolled when children were toddlers.

- Inclusion of children of all gestational ages $(16 \%$ 28-31 completed weeks' gestation at birth; $21 \%, 32-36$ weeks' gestation; $63 \%, \geq 37$ weeks' gestation)

- Video-recording of parent-child interaction in mealtime and play settings in the home and the laboratory.

- Participants from a single region (Central Ohio, USA).

- Preschool-age visits interrupted by COVID-19 pandemic.

related to children's weight gain and adiposity, although whether the child's weight is the cause or consequence of food parenting practices is unclear. ${ }^{23}$ The emotional context of parent-child interactions and how caregiver responses to infant's cues inform children's recognition and interpretation of hunger has long been linked to obesity. ${ }^{2-6}$ Epidemiological evidence suggests that risk for obesity is increased for young children who experience poor-quality emotional relationships with their parents, and these associations are not fully explained by differences in family socioeconomic position. ${ }^{78}$

Decades of research by developmental scientists also demonstrate that positive parent-child relationships support children's development of self-regulation. ${ }^{9}$ Prospective studies have suggested that children with lower self-regulation are more likely to be obese later in life, ${ }^{10}$ and children born preterm are at higher risk for deficits in selfregulation. ${ }^{11}$ Difficulties with self-regulation could explain how poor-quality parent-child interactions increase obesity risk, but this has not been established, and which aspects of self-regulation are involved is only beginning to be explored. ${ }^{1610-12}$ Furthermore, parenting and child self-regulation may interact as 
predictors of children's risk for obesity. ${ }^{13}$ Research in diverse cohorts is needed to ensure that potential heterogeneity in associations guide development of theory. ${ }^{6}{ }^{14}$ Parent-child interactions are complex, and childhood obesity prevention efforts are strengthened by the use of observational protocols to assess parent-child interactions broadly and across settings.

The extent to which parent-child interactions in the context of eating or mealtimes differ from how parents interact with their children in non-food settings has received surprisingly little research attention. An exception is a cross-sectional study of 21 mothers and their preschool-aged child who were observed in a laboratory setting during lunch and while completing a puzzle task. ${ }^{4}$ They report associations with child adiposity relative to patterns of mother-child interaction and conclude that children with higher weight experienced less responsive interactions in each context (ie, eating or play). ${ }^{4}$ In a published study of a cohort of infants at high risk due to maternal substance use, Kong et al reported that warm and positive interactions between mothers and infants during play were associated with children's lower body mass index trajectories into elementary school, but quality of mother-infant interactions assessed during feeding were not associated with children's body mass index trajectories. ${ }^{15}$

The quality of parent-child interaction in relation to child outcomes, such as obesity, has typically been assessed by coding parent and child behaviour as observed during a semi-structured play task. Yet, it is plausible that parentchild interactions differ between mealtime and playtime contexts. Observations of diverse families over time with consistent measurement of parent-child interactions across contexts can inform the development of obesity prevention strategies targeting young children.

Premature birth has significant and lasting impacts on parenting and the parent-child relationship. ${ }^{16}$ Preterm infants are smaller than their term peers for a given calendar age, and some struggle during the first few years of life to catch up to the growth of their peers born at term. ${ }^{17}$ Growth faltering is associated with poor outcomes, so clinical care for children born preterm often focuses on promoting growth. ${ }^{18}$ However, by school-age most children born preterm do catch up to be of similar size as children born at term. ${ }^{17}$ Despite continued monitoring of growth as part of clinical care for children born preterm, little research attention has been focused on preventing excess weight, an under-recognised consequence of growth-promoting behaviours that continue after adequate growth is achieved. Empirical research to assess the impact of preterm birth on food parenting practices beyond infancy is also limited.

Childhood obesity is an important public health problem that has resisted easy solutions despite substantial efforts. ${ }^{19} 20$ Children born preterm ( $<37$ weeks' gestation) have risks for obesity that are similar to those of children born full term, ${ }^{21}{ }^{22}$ and higher weight gain throughout the first year of life is linked to an increased risk for obesity in term ${ }^{2324}$ and preterm children. ${ }^{25}$ Obesity prevention efforts tailored to young children and their families hold promise,${ }^{26}{ }^{27}$ but there is a need for longitudinal research to understand the complex, reciprocal interactions through which parents create environments that shape children's development and obesity risk. ${ }^{1428}$ In particular, toddlers are understudied relative to infants and school-age children. This paper describes the design of the Play \& Grow Cohort, a longitudinal cohort study of diverse Ohio families with toddlers.

\section{COHORT DESCRIPTION}

Play \& Grow is a prospective cohort study of 300 caregiverchild dyads residing in Central Ohio in the Midwestern region of the USA. The primary goal of this study is to determine how the quality of parent-child interactions observed in mealtime and play settings in the home and laboratory impact changes in weight and risk for obesity as children get older and identify the aspects of child self-regulation that are involved. This report describes the recruitment of the cohort, outlines study protocols used during the toddler (18 months and 24 months of age) and preschool-age (36 and 42 months of age) visits, describes the sociodemographic and weight status profile of the cohort and the association of preterm birth with cohort characteristics. Data collection for the toddler phase of the study was completed in December 2019 and the preschool phase was completed in January 2021.

\section{Eligibility and recruitment}

Recruitment for the Play \& Grow Cohort began in November 2017. Families were recruited from two source populations using patient records maintained by Nationwide Children's Hospital (Columbus, Ohio, USA). Nationwide Children's Hospital is the only provider of subspecialty and emergency paediatric care in the region, and the major provider of paediatric primary care. Nationwide Children's Hospital electronic medical records identified children whose calendar age was $\geq 16.0$ and $<17.0$ months, and who had visited, at any point in their lives, a Nationwide Children's Hospital Urgent Care Center, or who had been a patient in a Nationwide Children's Hospital-affiliated neonatal intensive care unit on their preterm birth or been referred to the neonatology clinic for follow-up after a preterm birth. These two source populations (urgent care and neonatal intensive care unit/neonatology clinic) were used to ensure sociodemographic diversity of participants across gestational ages.

\section{Exclusion and inclusion criteria}

Participating children were required to be born a singleton, be $18 \pm 2$ months chronological age at study enrolment, have their gestational age available in their medical record or reported by their caregiver if not available in the medical record, live within 15 miles of the Nationwide Children's Hospital Main Campus in 
downtown Columbus with no family plans to move beyond that radius in the next 2 years; the child had to be able to communicate, self-feed and move around the room during play. Additionally, the caregiver needed to be the child's legal guardian, speak English with the child and participate in the child's meals on a regular basis. If two caregivers met these criteria, the primary caregiver was self-nominated and remained in that role across all visits. Participants were excluded if any of the following criteria were met: child deafness, child blindness, a caregiver or child food allergy, the child's recorded gestational age exceeded 42 weeks or the child was tube-fed or a patient for a clinical feeding disorder. Additionally, children born at term who had been patients in the neonatal intensive care unit were excluded. The rationale for this exclusion was the likelihood of severe clinical conditions associated with neonatal intensive care unit admission for term neonates. Children whose medical record suggested they met inclusion and exclusion criteria were screened by study staff, and eligibility was confirmed with caregivers prior to enrolment. However, after recruitment we determined that four children who were born at term and had short stays ( $<7$ days) at a Nationwide Children's Hospital neonatal intensive care unit as infants and had enrolled in the study. We decided to retain these children in the cohort because a review of their medical histories indicated that their stays in the neonatal intensive care unit were not attributed to a severe health condition and their overall development reflected that of a healthy, typically developing child.

\section{Sample selection and participant invitation}

Recruitment took place between November 2017 and May 2019. The Nationwide Children's Hospital database was queried monthly to generate a list of 100-150 children to attempt for recruitment; this included all age-eligible children whose gestational age was $<35$ completed weeks (approximately 30-40 children/month) and a random sample of children with unknown or later gestational ages at birth. In total, 2670 children were identified for potential recruitment (figure 1). Of these, 671 children were not invited to participate because research staff identified an exclusion in their medical record prior to contact $(\mathrm{n}=294)$, or because more children were eligible during a particular month than could be accommodated by the research team $(\mathrm{n}=377)$.

Of the 1999 children and their caregivers invited to participate, 300 enrolled. To initiate recruitment, study staff identified the child's legal guardian and contact information in the medical record. Recruitment began with a letter and a phone call 1 week later to gauge interest and further assess eligibility. If not reached during an initial call, contact attempts were repeated by phone, email or text message at varying times of the week and day. Eligible and interested families were scheduled to complete the informed consent process and enrol at the initial study visit. In total, 151 children and caregivers were deemed ineligible during a phone conversation with the caregiver,
198 caregivers actively declined to participate and 46 scheduled a visit but did not attend. Recruitment efforts continued with each family until the child reached 20 months of age. In total, 1304 caregivers and children were unresponsive to contact attempts: 1263 passively declined by not responding, and 41 were not locatable with the available contact information (figure 1). Recruitment was monitored by child sex and race and ethnicity to balance sociodemographic characteristics relative to children's gestational age. Our goal was to enrol a diverse cohort that included children of all gestational ages and overrepresented children who were born very preterm $(<32$ completed weeks).

\section{METHODS OF DATA COLLECTION}

The primary caregiver and child attended the initial study visit at the Nationwide Children's Hospital observational laboratory, gave written informed consent to participate in this longitudinal study consisting of four planned study visits over 2 years during the initial funding period (two in the laboratory and two in their home), each involving various video-recorded tasks. The toddler phase of the study included two visits separated by 6 months. The first visit coincided with enrolment and took place at the Nationwide Children's Hospital Center for Biobehavioral Health when children were 16-19 months' calendar age. The second study visit occurred in the participant's home when the child was approximately 24 months old. The preschool phase of the study began in June 2019 when children were 3 years old, and like the design of the toddler phase, included paired home and laboratory visits separated by 6 months. As will be described subsequently, the preschool phase of the study was impacted by the global COVID-19 pandemic.

\section{Patient and public involvement}

The study's research questions and protocols were developed from pilot studies also conducted with individuals affiliated with Nationwide Children's Hospital. Feedback was collected from participants at these pilot visits and used to help finalise the study's design. Although patients were not directly involved in the recruitment and conduct of the study, their well-being was considered in all aspects of the design and procedures. Results have been and will continue to be disseminated to study participants through periodic newsletters and multimodal communication overseen by Nationwide Children's Hospital (eg, website, social media, research blogs).

\section{Toddler phase overview}

Protocol for first visit

Caregivers completed a 45 min self-administered questionnaire on a tablet computer or on paper. Questions covered infant feeding practices (breast feeding, age at introduction of complementary foods), children's daily routines (eg, About Your Child's Eating ${ }^{29}$ ), temperament (Early Childhood Behavior Questionnnaire, Very Short 


\section{ASSESSED FOR ELIGIBILITY $(n=2670)$}

- NCH Urgent Care patients or NICU/Neonatology Clinic patients born preterm

- Calendar age 16.0 to $<17.0$ months

- Singleton gestation

- Residing within 15 -mile radius of $\mathrm{NCH}$
PRESCREENED, BUT NOT INVITED TO

PARTICPATE $($ Total $=671)$ :

1. INELIGIBLE $(n=294)$

- Multiple gestation $n=117$

- Feeding tube/disorder $n=52$

- Outside recruitment radius $n=24$

- Food Allergies $n=12$

- Ineligible for other reasons $n=89$

2. ELIGIBLE, BUT NOT RECRUITED $(n=377)$

- Due to pacing of enrollment $n=190$

- More eligible families then staff $n=187$

Invited to Participate

(Nov. 2017-May 2019) $n=1999$

Enrolled in Play \& Grow Study

(Dec. 2017-May 2019) $n=300$

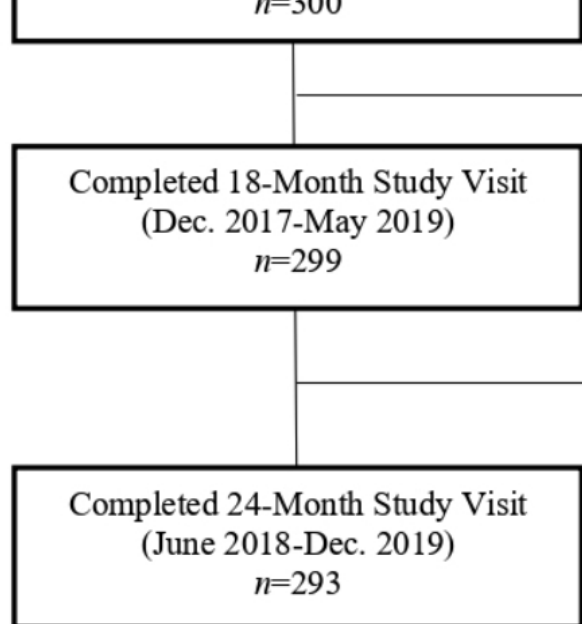

\section{CONTACTED, BUT NOT ENROLLED (Total=1699):}

1. DEEMED INELIGIBLE VIA PHONE $(n=151)$

- Food Allergies $n=42$

- Plans to move from the area $n=24$

- Multiple gestation $n=23$

- English not primary language $\quad n=17$

- Feeding tube/disorder $\quad n=2$

- Developmental delays $\quad n=6$

- Other (i.e. guardianship issues) $\quad n=37$

2. DECLINED TO PARTICIPATE VIA PHONE $(n=244)$

- Actively declined $n=198$

- Did not attend scheduled visit $\quad n=46$

3. UNRESPONSIVE TO CONTACT ATTEMPTS $(n=1304)$

- Passively declined $n=1263$

- Unable to locate $n=41$

DID NOT COMPLETE STUDY VISIT 1 (Total $=1)$

- Withdrew $n=1$

DID NOT COMPLETE STUDY VISIT 2 (Total= 6)

- Withdrew $n=2$

- Lost to follow up $n=1$
- Domestic guardianship issues $n=3$

Figure 1 Participant flow diagram. NCH, Nationwide Children's Hospital; NICU, neonatal intensive care unit. 
Form $^{30}$ ), development (Knowledge of Infant Development Short-Form ${ }^{31}$ ), caregiver health and relationships (depression and anxiety screeners ${ }^{32} 33$; Parenting Stress Index, Short Form $^{34}$ ) and household characteristics including food security (USDA Household Food Security Survey ${ }^{35}$ ). Study staff remained in the room to entertain the child while the caregiver completed the questionnaires and were available to answer caregiver questions.

Other components of the visit required $45 \mathrm{~min}$ and included anthropometric measurement of caregivers and toddlers (described inAnthropometric measurements) and a video-recording of parent-child interaction during a semi-structured play protocol. ${ }^{36}$ Following standardised procedures, the caregiver and child were invited to sit on a mat on the floor, and a staff member presented a set of developmentally appropriate toys (Fisher-Price Little People Lil' Movers Airplane, VTech Busy Learners Activity Cube and Sassy Block Set Zoomin' Train). Caregivers were asked "to play as you would at home if you had some free time," and were instructed to try to keep the child and themselves oriented towards the camera with faces and hands visible.
Staff monitored the parent-child interaction remotely from an adjacent room for the 10 min task duration. Videos were uploaded to a secure server. Recordings are observed and coded by independent teams of coders supervised and trained by an expert coder (BJZ). Coding teams are blind to all other aspects of the family's status and data and to our specific hypotheses. Interactions are coded (using a 7-point scale with $1=$ very uncharacteristic and $7=$ eery characteristic) for the following global dimensions of parenting behaviour: sensitivity, detachment, intrusive control, stimulation, warm positive regard and harsh negative regard as well as overall mutuality of the interaction. This coding scheme is well-validated and has been used for mothers and fathers at varying levels of education and income as well as across ethnicities. ${ }^{37}$ The visit concluded with a series of administrative tasks which included scheduling the home visit for a time convenient to the family around the child's second birthday, thanking the caregiver with a US $\$ 50$ gift-card, book and study-branded blanket for the child, and facilitating transportation (ie, parking validation or taxi) (figure 2).

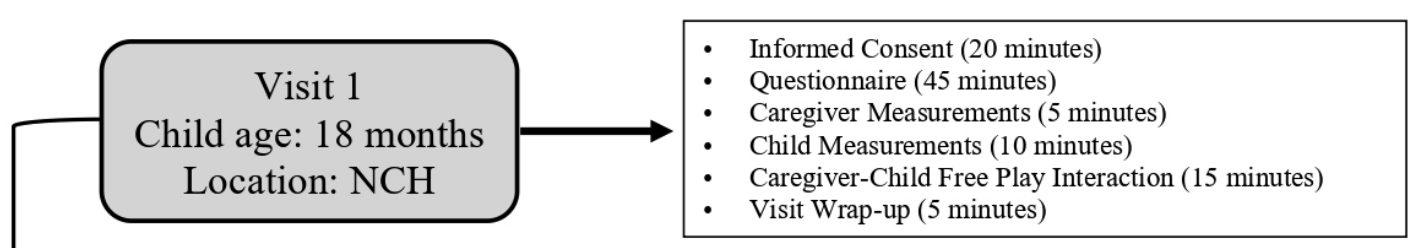

Toddler Phase

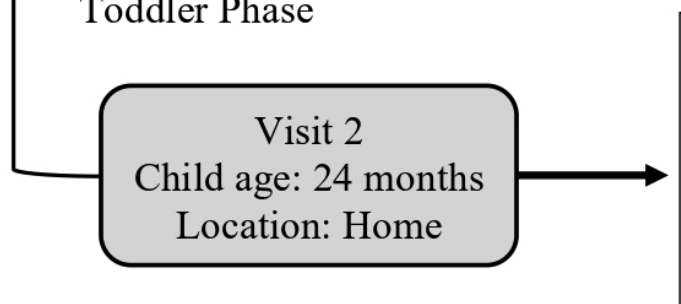

- Neighborhood Observations (10 minutes)

- Questionnaire (if not completed prior to visit) (30 minutes)

- Household Observations (throughout)

- Child Measurements (10 minutes)

- Picture Book Interaction (10 minutes)

- Puzzle Interaction (10 minutes)

- Visit Wrap up (10 minutes)

- $\quad$ Family Meal Interaction (30-60 minutes)

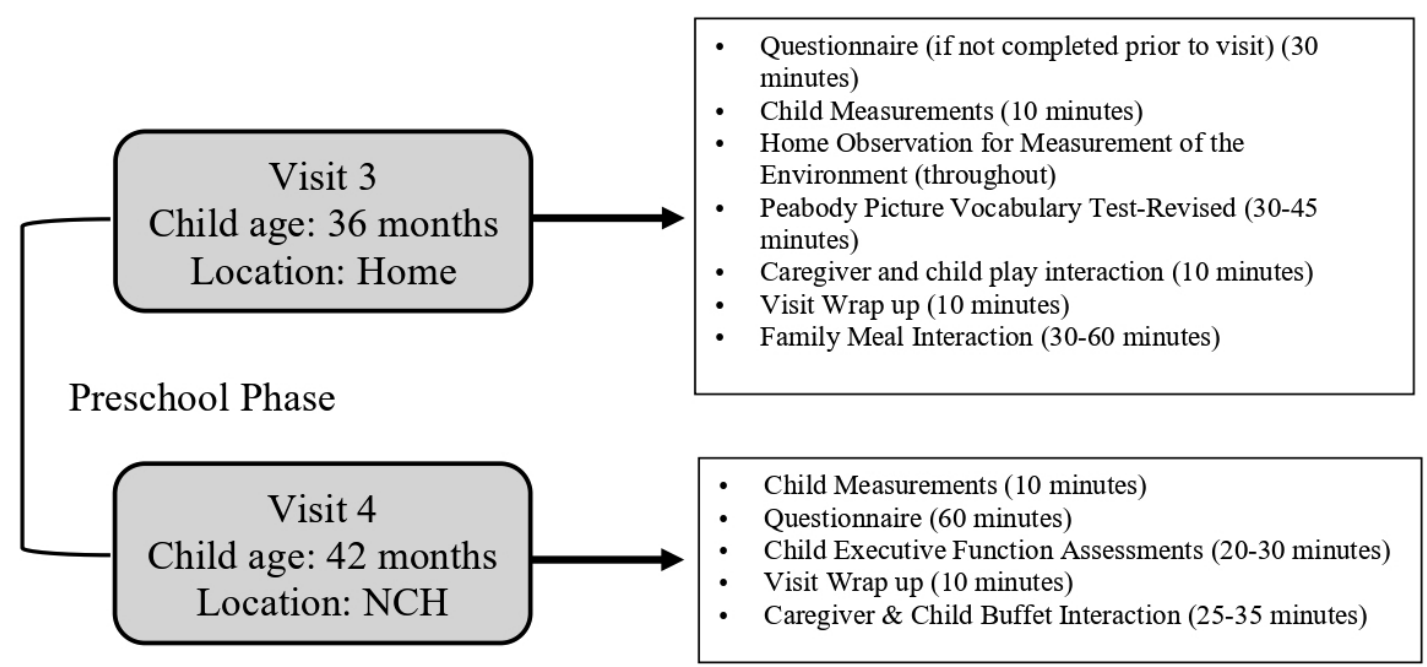

Figure 2 Overview of Play \& Grow study visits (2017-2021). NCH, Nationwide Children's Hospital. 


\section{Anthropometric measurements}

Research staff received standardised training in adult and paediatric anthropometric measurement, ${ }^{38}$ and were required to demonstrate accuracy and reliability in measurement of adult height and weight, and child weight, recumbent length and standing height before they were certified to measure participants. Absolute technical error of measurement was calculated for each trainee based on a minimum of 10 measurements, ${ }^{39}$ and if staff did not obtain acceptable levels of accuracy and precision, they repeated trainings and measurements until they demonstrated proficiency.

\section{Caregiver height and weight}

The height and weight of the primary caregiver was measured at study visit 1 . Height was measured to the nearest $0.1 \mathrm{~cm}$ using a Seca 284 stadiometer and weight was measured to the nearest $0.05 \mathrm{~kg}$ using a Seca 874 scale. Caregivers were dressed in light clothing without shoes. Equipment was calibrated prior to measurement. Height and weight measurements were taken three times according to a standardised procedure. ${ }^{38}$ If the primary caregiver was pregnant at the first study visit, height was recorded and weight measured at a subsequent visit. In addition, when the primary caregiver was not the biological mother $(n=20)$, we sought to measure the height and weight of the biological mother as well as the primary caregiver.

\section{Child weight and length}

After the caregiver was measured, the child was measured without shoes and in a clean diaper. Recumbent length was measured to the nearest $0.1 \mathrm{~cm}$ using a calibrated Seca 416 infantometer. To ensure accurate measurement, research staff and the caregiver assisted with positioning the child, and three repeated length measurements were recorded. Child weight was measured in triplicate to the nearest $0.05 \mathrm{~kg}$ with the same Seca 874 scale used with caregivers.

\section{Protocol for second visit}

The second study visit occurred in the family's home for approximately $90 \mathrm{~min}$ (figure 2) and was scheduled for \pm 1 week surrounding the child's second birthday. However, to maximise retention, caregivers were accommodated in the timing of the visit, and we rescheduled visits until the child was 30 months old. Trained staff members completed the home visits in teams of two. A component of the visit was video-recording of a typical family meal. The primary caregiver was asked to think about the child's perspective in defining the timing (lunch or dinner) of the meal and which family members to invite. Study staff scheduled the visit in coordination with the caregiver so that activities aligned with the child's routine. Any additional family members who participated in visit provided informed consent.

Caregivers were invited to complete a $30 \mathrm{~min}$ selfadministered questionnaire prior to the visit which assessed child and family routines (eg, sleep, screentime), feeding strategies (Feeding Strategies Questionnaire ${ }^{40}$ ), perceived neighbourhood safety ${ }^{41}$ as well as multiple aspects of parent physical (eg, sleep, activity, diet), social (eg, emotion-related beliefs ${ }^{42}$ ) and emotional health and well-being (eg, Self-Compassion Scale, Short Form). ${ }^{43}$ Prior to and during the home visit, staff conducted a qualitative and quantitative assessment of neighbourhood and household conditions. The methodology was adapted from existing instruments focused on neighbourhood and home environments, ${ }^{41-46}$ and included observations of housing quality and maintenance, noise, safety and amenities. A detailed description has been published. ${ }^{47}$ The neighbourhood was assessed for approximately 10 min prior to the start of the visit, and the home environment was observed throughout the visit (figure 2).

Children's standing height and weight were measured in triplicate using a portable Seca 213 Stadiometer and a Seca 874 scale. Each instrument was calibrated, and staff were trained to place them on a flat, level surface. The child was dressed in light clothing without shoes. The weight of the primary caregiver and/or the biological mother was measured during the home visit as needed.

Parent-child interactions were video-recorded in the context of play, reading and a typical family meal. Research staff positioned the camera for each task on a tripod and ensured that the child and primary caregiver could be viewed on the videos facing forward or in profile. Parentchild play and reading interactions were video-recorded in two $10 \mathrm{~min}$ sessions; the first used a wordless picture book, ${ }^{48}$ and the second used three puzzles that ranged in difficulty. We concluded the visit by video-recording the child's typical family meal (dinner for $53 \%$ and lunch for $47 \%$ of families). Caregivers selected items for a family meal from a Subway Restaurants menu and staff brought the order to the home visit, but $13 \%$ elected to prepare their own meal instead. Research staff set up the camera to ensure that the child and primary caregiver's face and hands were visible (at least in profile) and to include other consented family members in the video as feasible. The camera was placed on a tripod and set-up at the start of the visit after asking the primary caregiver to identify the likely seating arrangements for the meal. The camera was turned on when the caregiver began preparing the family meal, was checked once for placement after the child began eating and was allowed to run for $25 \mathrm{~min}$ after the child's first bite or until the caregiver indicated that the meal was finished (whichever came first). Videorecordings of each of the three parent-child interaction videos (play, book, meal) were uploaded to a secure server for observational coding.

\section{Preschool phase overview}

The protocol for the preschool phase of the study included two study visits. Data collection for the 36-month visit, in the child's home, began in June 2019. Data collection for the 42-month visit, in the NCH biobehavioral laboratory, began in December 2019. These study visits included 


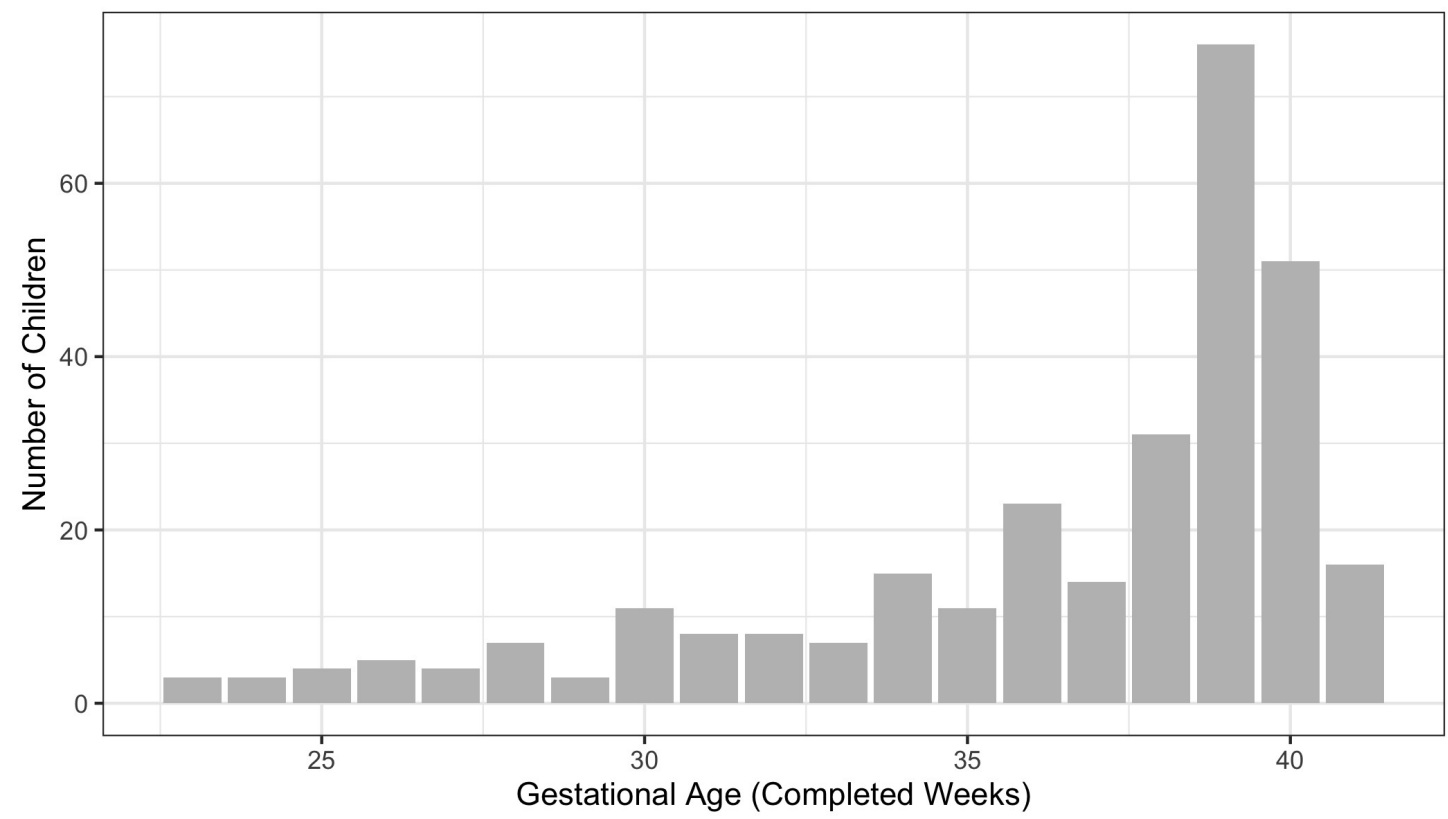

Figure 3 Distribution of gestational age (weeks' completed gestation at birth).

video-recordings of parent-child interaction in mealtime and non-mealtime settings, and measurement of child growth and development across multiple domains. Visits were targeted for plus or minus one-week of the child's age. The COVID-19 pandemic impacted the preschool phase study visits.

\section{Components of third visit}

The third visit (36 months) was like the second visit (24 months). It took place in the home and was similar in terms of duration ( $90 \mathrm{~min})$, accommodations for retention and study staff presence, training and oversight. Major activities included caregiver questionnaires, anthropometric measurements of the child, assessment of child cognitive development, observation of the home environment and video-recording of parent-child interaction during play and in a family meal (figure 2).

Prior to the visit, caregivers were invited to complete a 30 min self-administered questionnaire. The questionnaire included items to assess child sleep routines, activity and sedentary behaviour, and multiple domains of parental feeding practices. ${ }^{4-52}$ Other parent-report instruments included in the questionnaire were the Confusion, Hubbub and Order Scale, ${ }^{46}$ Parenting Daily Hassles Scale, ${ }^{53}$ Dyadic Adjustment Scale ${ }^{54}$ and the Strengths and Difficulties Scale. ${ }^{55}$ Caregivers also completed the Behavior Rating Inventory of Executive Function-Preschool Version (BRIEF-P) ${ }^{56}$ during the home visit.

The protocol for the third study visit was like the second visit relative to procedures for video-recording a typical family meal. Other components of the third visit are described next. While caregivers were completing the BRIEF-P (and other questionnaires if they had not completed them before the visit), one trained staff member completed the Home Observation for Measurement of the Environment-Short Form. ${ }^{57}$ The second trained staff member measured the child's height and weight as previously described, and administered the Peabody Picture Vocabulary Test-Revised (PPVT-5) ${ }^{58}$ to assess the child's receptive vocabulary as a measure of early cognitive ability. Following these activities, the child and caregiver were video-recorded playing with a standardised set of developmentally appropriate toys (barn with animals, alphabet puzzle, picture book $^{59}$ ) for $10 \mathrm{~min}$. Research staff positioned the camera on a tripod and ensured that the child and primary caregiver could be viewed on the videos facing forward or in profile. The visit concluded with video-recording of a typical family meal using the same protocol as at the second visit.

Components of fourth visit

The fourth visit occurred in the laboratory at Nationwide Children's Hospital. It was similar in length to the first study visit and administered by two trained staff. The child's height and weight were measured with the instruments and protocol used during the second and third visits. Additional components of the visit were caregiver questionnaires, assessment of child executive function and video-recording of parent-child interaction during a standardised buffet lunch.

Caregivers completed self-administered questionnaires before and during the visit. These included the Child Eating Behavior Questionnaire, ${ }^{60}$ Dutch Eating Behavior Questionnaire, ${ }^{61}$ Parenting Stress Index, Short Form, ${ }^{34}$ Child Behavior Checklist for Ages 11/2-5, ${ }^{62}$ Difficulties in Emotion Regulation Scale, ${ }^{63}$ Childhood Trauma Questionnaire ${ }^{64}$ and the Block Questionnaire for Ages 2-7 (Kids 2-7 FFQ). ${ }^{65}$

While the caregiver was completing the questionnaires in a separate room, trained staff administered a series of standardised executive function tasks with the child. Assessments were video-recorded and the total time spent on the executive function assessments was between 20 
Table 1 Child, caregiver and household characteristics

\begin{tabular}{ll}
\hline Child characteristics & N (\%) \\
\hline Gestational age at birth & \\
$37-41$ completed weeks (term) & $188(63 \%)$ \\
$<37$ completed weeks (preterm) & $112(37 \%)$ \\
Sex &
\end{tabular}

\begin{tabular}{|c|c|}
\hline Male & $170(57 \%)$ \\
\hline Female & $130(43 \%)$ \\
\hline \multicolumn{2}{|l|}{ Race/Ethnicity } \\
\hline Non-Hispanic white & $137(46 \%)$ \\
\hline Non-Hispanic black & $105(35 \%)$ \\
\hline Non-Hispanic other (includes multiple races) & $35(12 \%)$ \\
\hline Hispanic & $23(8 \%)$ \\
\hline Caregiver characteristics & $\mathbf{N}(\%)$ \\
\hline \multicolumn{2}{|l|}{ Relationship to child } \\
\hline Biological mother & $280(93 \%)$ \\
\hline Biological father & $15(5 \%)$ \\
\hline Other* & $5(2 \%)$ \\
\hline \multicolumn{2}{|l|}{ Age (years) at enrolment } \\
\hline 18 to $<21$ & $8(3 \%)$ \\
\hline 21 to $<25$ & $46(15 \%)$ \\
\hline 25 to $<30$ & $62(21 \%)$ \\
\hline 30 to $<35$ & $98(33 \%)$ \\
\hline 35 to $<40$ & $62(21 \%)$ \\
\hline 40 or older & $23(8 \%)$ \\
\hline \multicolumn{2}{|l|}{ Race/ethnicity } \\
\hline Non-Hispanic white & $158(53 \%)$ \\
\hline Non-Hispanic black & $111(37 \%)$ \\
\hline Non-Hispanic other (includes multiple races) & $18(6 \%)$ \\
\hline Hispanic & $13(4 \%)$ \\
\hline \multicolumn{2}{|l|}{ Marital status } \\
\hline Married & $162(55 \%)$ \\
\hline Living with partner & $62(21 \%)$ \\
\hline Single/Never married & $58(20 \%)$ \\
\hline Othert & $15(5 \%)$ \\
\hline \multicolumn{2}{|l|}{ Education level } \\
\hline High school or less & $70(23 \%)$ \\
\hline Some college or associate's degree & $103(34 \%)$ \\
\hline Bachelor's degree & $67(22 \%)$ \\
\hline Graduate degree & $59(20 \%)$ \\
\hline Household characteristics & N (\%) \\
\hline \multicolumn{2}{|l|}{ Annual household income (US\$) } \\
\hline$<20000$ & $78(26 \%)$ \\
\hline 20000 to $<50000$ & $89(30 \%)$ \\
\hline 50000 to $<90000$ & $57(19 \%)$ \\
\hline 90000 or more & $73(25 \%)$ \\
\hline \multicolumn{2}{|l|}{ Household food security $\ddagger$} \\
\hline High food security & $206(69 \%)$ \\
\hline Marginal food security & $42(14 \%)$ \\
\hline
\end{tabular}

Continued

\section{Table 1 Continued}

\begin{tabular}{lc} 
Low food security & $37(12 \%)$ \\
Very low food security & $14(5 \%)$ \\
Household occupants & Mean (SD) \\
Number of adults & $2.0(0.63)$ \\
Number of children & $2.2(1.4)$ \\
\hline
\end{tabular}

Percentages may not total to $100 \%$ due to rounding.

Information was missing for marital status $(n=3)$, caregiver education $(n=1)$, household income $(n=3)$ and household food security $(n=1)$.

*Includes adoptive mother $(n=3)$, grandmother $(n=1)$, other, nonrelative $(n=1)$.

†Includes "partner and I not living together" $(n=4)$, separated $(n=5)$ and divorced $(\mathrm{n}=6)$.

\#Food security was assessed using the 18-item USDA scale.

and $30 \mathrm{~min}$. The Flanker and Dimensional Change Card Sort components of the NIH toolbox were administered on a tablet computer to assess inhibitory control, attention and task shifting. ${ }^{66}$ We used a Gift Bag and Snack Delay protocol to assess delay of gratification in non-food and food settings, and a frustration task (Locked Box) to assess emotion-regulation. ${ }^{9}$

The visit concluded with video-recording of a buffetstyle lunch designed to observe parent-child mealtime interactions in a laboratory setting. The meal included a large variety and quantity of foods prepared by the NCH Nutrition Services Department and study staff. A standardised protocol was used to allow for comparisons of parent-child mealtime interactions holding constant aspects of the environment related to sociodemographic characteristics. In contrast to observations in the home, we can ensure that the laboratory environment is comparable and not impacted by other potentially confounding factors such as presence of other children/family members, pets, time pressures, having the television on or other media use. The room contained two cameras. A table held a chafing dish containing macaroni and cheese, breaded chicken fingers and French fries. The buffet table also included an assortment of meats, cheeses, bread, vegetables, fruit, salads, condiments, beverages, snacks and desserts. The foods spanned the spectrum of nutritional quality and we purposefully included both foods that would be familiar and unfamiliar to children and caregivers. The buffet was designed to elicit food parenting behaviours that might not be seen in a home environment. For example, multiple appealing foods of minimal nutritional value (eg, candies, cookies, potato chips) were easily accessible to the child on two "coffee tables' at either end of the main food table. A separate square dining table with places set for the child and caregiver was positioned facing a second camera. The child and caregiver were brought by a staff member to the private room in which the buffet had been laid out. They were instructed that the food was prepared fresh for them and that they could help themselves to anything from the buffet. The staff member then monitored the dyad from 


\begin{tabular}{|c|c|}
\hline Child & $\mathbf{N}(\%)$ \\
\hline \multicolumn{2}{|l|}{ Birth weight, $\mathrm{g}^{*}$} \\
\hline$<1000$ & $20(7 \%)$ \\
\hline 1000 to $<2000$ & $41(14 \%)$ \\
\hline 2000 to $<3000$ & $76(26 \%)$ \\
\hline 3000 to $<4000$ & $144(48 \%)$ \\
\hline$\geq 4000$ & $17(6 \%)$ \\
\hline Visit 1 anthropometric measurements & Mean (SD) \\
\hline Length, cm & $79.7(3.7)$ \\
\hline Weight, kg & $10.9(1.4)$ \\
\hline WHO BMI-for-age z-score $\dagger$ & $0.75(0.98)$ \\
\hline WHO BMI-for-age z-score category $†$ & $\mathbf{N}(\%)$ \\
\hline Underweight (BMI z-score <-2) & $2(0.7 \%)$ \\
\hline Healthy weight (BMI $z$-score -2 to $<1$ ) & $180(60 \%)$ \\
\hline $\begin{array}{l}\text { Possible overweight (BMI z-score } 1 \\
\text { to }<2 \text { ) }\end{array}$ & $86(29 \%)$ \\
\hline Overweight and obesity (BMI z-score $\geq 2$ ) & $31(10 \%)$ \\
\hline Caregiver & Mean (SD) \\
\hline \multicolumn{2}{|l|}{ Anthropometric measurements } \\
\hline Height (m) & $1.64(0.072)$ \\
\hline Weight (kg)‡ & 82.7 (23.3) \\
\hline BMI $\left(\mathrm{kg} / \mathrm{m}^{2}\right) \ddagger$ & $30.6(7.9)$ \\
\hline Weight status $\ddagger$ & $\mathbf{N}(\%)$ \\
\hline Underweight (BMI <18.5) & $6(2 \%)$ \\
\hline Healthy weight (BMI 18.5 to <25) & $79(28 \%)$ \\
\hline Overweight (BMI 25 to <30) & $63(22 \%)$ \\
\hline Obesity (BMI $\geq 30$ ) & $139(48 \%)$ \\
\hline
\end{tabular}

$\mathrm{N}=299$; excludes one caregiver-child dyad who did not complete visit 1 . Percentages may not total to $100 \%$ due to rounding. *Birth weight was not available for one child.

†Children's age was adjusted for preterm birth if children were born at <37 completed weeks' gestation. However, results were similar using unadjusted calendar age to calculate WHO BMIfor-age z-scores. Mean (SD)=0.80 (0.97); BMl z-score $<-2(n=1)$, BMl $z$-score -2 to $<1(n=179)$, BMI $z$-score 1 to $<2(n=85)$, BMI $z$-score $\geq 2(n=34)$. BMl-for-age $z$-score cut points and category labels as recommended by $\mathrm{WHO}{ }^{67}$

$\ddagger$ Excludes caregivers $(n=12)$ who were pregnant or not measured at visit 1

BMI, body mass index.

a separate room and returned after 25 min or when it was apparent that they were done eating.

\section{Sociodemographic characteristics}

The 300 children (57\% male) in the Play \& Grow Cohort were born between June 2016 and December 2017 and were enrolled in the study at a mean (SD) (IQR) calendar age of $18.2(0.70)(0.85)$ months. Children were born at gestational ages ranging 23-41 weeks' completed gestation (figure 3). The proportion of children born preterm $(<37$ weeks) was $37 \%(\mathrm{n}=112)$ and this included 48 children born extremely or very preterm ( $<32$ weeks). The primary caregiver was typically the biological mother $(93 \%)$. The cohort includes diversity in child and caregiver race and ethnicity with a majority of children identified by their caregivers as having non-white race or ethnicity (table 1). Caregiver education ranged from high school degree or less (23\%) to graduate degree $(20 \%)$. Age of caregivers ranged from 18 to 54 years with a mean (SD) of 31 (6) years. Most caregivers (76\%) were married or living with a partner. The number of children in the household at the time of the first study visit ranged from 1 to $10 ; 36 \%$ of children were the only child in the household. Household income varied widely; $26 \%$ had annual household incomes below US\$20 000 and 25\% had incomes above US $\$ 90000$. Household food security was low or very low for $17 \%$ of participants (table 1 ).

\section{Child and caregiver weight status}

At birth, children weighed between 520 and 5310 g; $21 \%$ were born at $<2000 \mathrm{~g}$ (table 2). At enrolment, children weighed a mean (SD) of 10.9 (1.4) $\mathrm{kg}$ and were 79.7 (3.7) $\mathrm{cm}$ in length. We calculated body mass index as weight $(\mathrm{kg})$ divided by the square of height $(\mathrm{m})$. Triplicate height and weight measurements were averaged, or if two measurements were identical, that value was used. We used body mass index to categorise caregiver weight status: underweight (body mass index $<18.5 \mathrm{~kg} / \mathrm{m}^{2}$ ), healthy weight (body mass index $\geq 18.5$ and $<25 \mathrm{~kg} / \mathrm{m}^{2}$ ), overweight (body mass index $\geq 25$ and $<30 \mathrm{~kg} / \mathrm{m}^{2}$ ) and obesity (body mass index $\geq 30 \mathrm{~kg} / \mathrm{m}^{2}$ ). Children's weight status was defined relative to the WHO Child Growth Standards. ${ }^{67}$ Sex-specific body mass index-for-age z-scores were calculated using child measurements of weight and recumbent length at visit 1 and weight and standing height at visit 2. Age at measurement was calculated using date of birth, and if children were born before 37 completed weeks' gestation, age was adjusted for prematurity. Body mass index-for-age z-scores ranged from -2.36 to 3.47; more children $(\mathrm{n}=31)$ had high body mass index-for-age $z$-scores (above 2) than children $(n=2)$ who had low body mass index-for-age z-scores (below-2). These cut points have been recommended by WHO for classifying overweight and underweight in children. ${ }^{67}$ Caregiver anthropometric measurements and weight status are shown in table 2. Almost half (48\%) of caregivers had a body mass index $\geq 30 \mathrm{~kg} / \mathrm{m}^{2}$.

\section{Comparison of sociodemographic characteristics by child gestational age}

Children born preterm ( $<37$ weeks) were similar to children born at term ( $\geq 37$ weeks) with respect to most child and caregiver characteristics (table 3). However, although not statistically significant $\left(\mathrm{p}=0.09\right.$ from $\left.\chi^{2}\right)$, the sex ratio among term children includes more boys than girls (table 3 ). Children born preterm were smaller as toddlers than children born at term (mean difference of $2.3 \mathrm{~cm}, 0.8 \mathrm{~kg}$ and 0.3 units for length, weight and WHO BMI-for-age z-score, respectively). However, 
Table 3 Comparison of child and caregiver characteristics by gestational age at birth

\section{Full term:}

$\geq 37$ weeks

\begin{tabular}{|c|c|c|c|c|c|}
\hline Child characteristics & $\mathbf{N}$ & Per cent (\%) & $\mathbf{N}$ & Per cent (\%) & P value* \\
\hline \multicolumn{6}{|l|}{ Sex } \\
\hline Male & 114 & 61 & 56 & 50 & 0.09 \\
\hline Female & 74 & 39 & 55 & 50 & \\
\hline \multicolumn{6}{|l|}{ Race/Ethnicity } \\
\hline Non-Hispanic white & 93 & 49 & 44 & 40 & 0.33 \\
\hline Non-Hispanic black & 60 & 32 & 45 & 41 & \\
\hline $\begin{array}{l}\text { Non-Hispanic other (includes multiple } \\
\text { races) }\end{array}$ & 22 & 12 & 12 & 11 & \\
\hline Hispanic & 13 & 7 & 10 & 9 & \\
\hline Anthropometric measurements at visit 1 & Mean (SD) & & Mean (SD) & & \\
\hline Length, $\mathrm{cm}$ & $80.5(3.3)$ & & $78.2(3.9)$ & & $<0.0001$ \\
\hline Weight, kg & $11.2(1.3)$ & & $10.4(1.6)$ & & $<0.0001$ \\
\hline WHO BMI-for-age z-score $†$ & $0.86(0.90)$ & & $0.56(1.1)$ & & 0.009 \\
\hline \multicolumn{6}{|l|}{ WHO BMI-for-age z-score category $\dagger$} \\
\hline Underweight (BMl z-score <-2) & 0 & 0 & 2 & 2 & 0.23 \\
\hline Healthy weight (BMI z-score -2 to $<1$ ) & 111 & 59 & 69 & 62 & \\
\hline $\begin{array}{l}\text { Possible overweight (BMI z-score } 1 \\
\text { to }<2 \text { ) }\end{array}$ & 58 & 31 & 28 & 25 & \\
\hline Overweight and obesity (BMI z- & 19 & 10 & 12 & 11 & \\
\hline
\end{tabular}

Preterm:

Preterm:

\begin{tabular}{|c|c|c|c|c|c|}
\hline Caregiver characteristics & $\mathbf{N}$ & Per cent $(\%)$ & $\mathbf{N}$ & Per cent (\%) & $P$ value \\
\hline \multicolumn{6}{|l|}{ Relationship to child } \\
\hline Biological mother & 174 & 93 & 105 & 95 & 0.22 \\
\hline Biological father & 12 & 6 & 3 & 3 & \\
\hline Otherf & 2 & 1 & 3 & 3 & \\
\hline \multicolumn{6}{|l|}{ Race/Ethnicity } \\
\hline Non-Hispanic white & 106 & 56 & 52 & 47 & 0.34 \\
\hline Non-Hispanic black & 63 & 34 & 48 & 43 & \\
\hline $\begin{array}{l}\text { Non-Hispanic other (includes multiple } \\
\text { races) }\end{array}$ & 10 & 5 & 7 & 6 & \\
\hline Hispanic & 9 & 5 & 4 & 4 & \\
\hline \multicolumn{6}{|l|}{ Marital status } \\
\hline Married & 106 & 56 & 56 & 50 & 0.28 \\
\hline Living with partner & 36 & 19 & 26 & 23 & \\
\hline Single/Never married & 33 & 18 & 25 & 23 & \\
\hline Other & 12 & 6 & 3 & 3 & \\
\hline \multicolumn{6}{|l|}{ Education level } \\
\hline High school or less & 38 & 20 & 32 & 29 & 0.10 \\
\hline Some college or Associate's degree & 62 & 33 & 41 & 37 & \\
\hline Bachelor's degree & 44 & 23 & 23 & 21 & \\
\hline \multirow[t]{2}{*}{ Graduate degree } & 44 & 23 & 15 & 14 & \\
\hline & Mean (SD) & & Mean (SD) & & $P$ value \\
\hline Caregiver age (years) & $30.7(5.9)$ & & $31.1(6.5)$ & & 0.61 \\
\hline Caregiver BMI & $30.2(8.1)$ & & $31.2(7.5)$ & & 0.32 \\
\hline
\end{tabular}


Table 3 Continued

\begin{tabular}{|c|c|c|c|c|c|}
\hline & $\begin{array}{l}\text { Full term: } \\
\geq 37 \text { weeks }\end{array}$ & & $\begin{array}{l}\text { Preterm: } \\
<37 \text { weeks }\end{array}$ & & \\
\hline Household characteristics & $\mathbf{N}$ & Per cent (\%) & $\mathbf{N}$ & Per cent (\%) & $P$ value \\
\hline$<20000$ & 40 & 21 & 38 & 35 & 0.02 \\
\hline 20000 to $<50000$ & 53 & 28 & 36 & 33 & \\
\hline 90000 or more & 51 & 27 & 22 & 20 & \\
\hline \multicolumn{6}{|l|}{ Household food security§ } \\
\hline High food security & 139 & 74 & 67 & 60 & 0.007 \\
\hline Marginal food security & 27 & 14 & 15 & 14 & \\
\hline Low food security & 14 & 7 & 23 & 21 & \\
\hline Number of children in household & $2.2(1.4)$ & & $2.2(1.4)$ & & 0.94 \\
\hline
\end{tabular}

Information missing for caregiver marital status $(n=2)$, household income $(n=2)$.

Percentages are column percentages and may not total to $100 \%$ due to rounding.

${ }^{*} \mathrm{P}$ values from $\chi^{2}$ (categorical variables) and t-tests (continuous variables).

†BMI-for-age z-score cut points and category labels as recommended by WHO ${ }^{67}$ Children's age was adjusted for preterm

birth if children were born at $<37$ completed weeks' gestation.

flncludes adoptive mother $(n=3)$, grandmother $(n=1)$, other, non-relative $(n=1)$.

§Food security was assessed using the 18-item USDA scale.

BMI, body mass index.

the distribution of child weight status categories did not differ statistically significantly between preterm and term children $(p=0.23)$, and the prevalence of overweight and obesity ( $10 \%$ of term and $11 \%$ of preterm) was similar in each group (table 3). Children born preterm were more likely to live in households with annual incomes below US $\$ 20,000$ and less likely to live in food secure households (table 3).

\section{Participant retention and completeness of data}

One caregiver-child dyad did not complete the first study visit and was not subsequently contacted. Thus, the sample size for analyses of data collected when children were 18 months old is 299. Data collection for the 24-month study visit began in June 2018 and concluded in December 2019. Of the 299 eligible caregiver-child dyads, 293 (98\%) participated in the second study visit. Children were a mean (SD) calendar age of $24.0(0.9)$ months. Home visits were completed with 284 families and an additional 9 families completed questionnaires online (figure 1). We measured the height and weight (non-pregnant) of all but five of the caregivers enrolled in the study and obtained the measured height and weight (non-pregnant) of the child's biological mother for $93 \%$ of the cohort.

The COVID-19 pandemic impacted the preschool phase of the study. Face-to-face data collection was suspended on 13 March 2020. At that point, 130 families had completed the 36-month visit and 44 dyads had completed the 42-month visit. In response to uncertainty about when research activities would again become feasible, it was decided in the summer of 2020 to focus on collecting the caregiver-report measures for the remaining 36-month visits remotely; in total 278 (93\%) of dyads have complete or partial data for the 36-month visit. The 42-month visit was paused between March and September 2020 with the intention to resume in-person study visits when permitted. We modified the protocol for the 42-month laboratory visit to reduce contact between research staff and participants and six dyads completed the study visit in fall of 2020 before a resurgence of COVID-19 again required cessation of face-to-face research and a transition to caregiver-response questionnaires. The protocol was also modified to ask caregivers to report on the impact of the pandemic on their own, their child's and their family's experiences. Pending funding, subsequent study visits are planned with this cohort as the children become school-age.

\section{Findings to date}

Sullivan et al analysed cross-sectional data from the first study visit to investigate the extent to which the caregiver's level of knowledge about typical infant and child development was associated with their well-being. ${ }^{68}$ Parents lacking knowledge of typical child development could hold expectations for their own child's behaviour which, if 
unrealistic, may impact their own level of stress or mental health. In our cohort, in alignment with prior research, caregivers' knowledge of typical child development was positively correlated with their overall education level and age, but we did not observe a relationship between knowledge of child development and any of the three aspects of well-being we investigated. ${ }^{68}$ Krupsky et al conducted a mixed-methods analysis of household chaos as observed during the second visit. ${ }^{47}$ Chaos has been found to have implications for child health and obesity, and an exploratory factor analysis examined the underlying structure of environmental and household chaos, and a thematic content analysis of ethnographies provided preliminary construct validity for these indicators of chaos. Evidence for a multifactor structure for chaos was found that included disorganisation and noise, and these constructs were associated with indicators of socioeconomic disadvantage (lower educational attainment and household income) ${ }^{47}$ Khalsa et al analysed correlates of parenting stress at the first study visit. ${ }^{69}$ Higher parenting stress was associated with higher levels of caregiver depressive symptoms, but levels of parenting stress were similar regardless of caregiver or child sociodemographic characteristics, gestational age at birth and child temperament. ${ }^{69}$

\section{Strengths and limitations}

Strengths of the Play \& Grow Cohort include direct observation and video-recording of parent-child interaction during mealtime and non-mealtime contexts in the home and laboratory setting over time. Video-recordings allow objective coding of parent and child behaviours. These video-recordings will allow for robust exploration of research questions focused on diverse families with toddlers. A further strength of the cohort was that participant recruitment was by invitation which avoids biases associated with participant self-selection. The cohort includes children across the full range of viable gestational ages (see figure 3), including 112 children born preterm. Children born prematurely are not immune to the problem of childhood obesity; their rates of obesity by school age are like children born at term ${ }^{1721}$ and preterm birth increases risk for cardiometabolic disease in later life. ${ }^{70}$ We aimed to recruit 90 children who were born very preterm $(<32$ weeks') and were only able to recruit half this many $(n=48)$. We believe that our sampling frame provided good coverage of the population of these children residing in Central Ohio because NCH is the predominant neonatal intensive care unit provider in the region. Therefore, to enrol more children born very preterm, it would have been necessary to expand our geographic area or lengthen the time for recruitment. Other limitations include the restricted geographic area from which our cohort was identified. However, Columbus Ohio is the 14th-most populous city in the USA, and demographically reflective of the USA at large. ${ }^{71}$

\section{Collaboration}

Multidisciplinary collaborations are ongoing. The research team is open to additional collaborations particularly with researchers interested in how early childhood psychosocial exposures relate to children's and families' outcomes in later life. The Play \& Grow Cohort is diverse and will contribute knowledge about the dynamics of family mealtime interactions over time in families with young children. We are coding parent-child interaction during mealtimes at 24, 36 and 42 months to assess stability and change in the emotional climate of family meals and better understand the range of mealtime experiences and parenting practices to which contemporary toddlers and preschool-age children are exposed. Findings will inform obesity prevention efforts to help parents create and maintain routines and home environments and engage in positive relationships with their young children to foster healthy growth and development.

Acknowledgements We are grateful for the participation of the families and the contributions of the research staff without whom this project would not have been possible. In particular, we acknowledge the work of Jacqueline Sullivan, Grace Pelak and Kathryn Krupsky who participated in data collection, protocol development, staff training, management and quality control. We thank Kristin Howard for assistance with analysis and creation of tables and figures.

Contributors SEA, BJZ, SAK and RA designed the study, provided oversight and obtained funding. AP managed the recruitment and data collection and drafted the manuscript. RA and SEA conducted analyses. All authors contributed to writing and revising of the paper and approved the final submission. SEA accepts full responsibility for the work and/or the conduct of the study, had access to the data, and controlled the decision to publish.

Funding This work was supported by the National Institute of Diabetes and Digestive and Kidney Diseases of the National Institutes of Health under award number R01DK108969 and supported by the National Centre for Advancing Translational Research of the National Institutes of Health under award number CTSA Grant UL1TR002733.

Disclaimer The content is solely the responsibility of the authors and does not necessarily represent the official views of the National Institutes of Health.

Competing interests None declared.

Patient and public involvement Patients and/or the public were involved in the design, or conduct, or reporting, or dissemination plans of this research. Refer to the 'Methods' section for further details.

Patient consent for publication Consent obtained directly from patient(s)

Ethics approval This study involves human participants and was approved by Nationwide Children's Hospital (IRB16-00826). Participants gave informed consent to participate in the study before taking part.

Provenance and peer review Not commissioned; externally peer reviewed.

Data availability statement Data are available upon reasonable request. The datasets generated and/or analysed during the current study are not publicly available unless approved by the Institutional Review Board overseeing this study but may be available from the corresponding author on reasonable request.

Open access This is an open access article distributed in accordance with the Creative Commons Attribution Non Commercial (CC BY-NC 4.0) license, which permits others to distribute, remix, adapt, build upon this work non-commercially, and license their derivative works on different terms, provided the original work is properly cited, appropriate credit is given, any changes made indicated, and the use is non-commercial. See: http://creativecommons.org/licenses/by-nc/4.0/.

ORCID iD

Sarah E Anderson http://orcid.org/0000-0002-8886-0392

\section{REFERENCES}

1 Fiese $\mathrm{BH}$, Bost KK. Family ecologies and child risk for obesity: focus on egulatory processes. Fam Relat 2016;65:94-107. 
2 Spill MK, Callahan EH, Shapiro MJ, et al. Caregiver feeding practices and child weight outcomes: a systematic review. Am J Clin Nutr 2019;109:990S-1002.

3 Vaughn AE, Ward DS, Fisher JO, et al. Fundamental constructs in food parenting practices: a content map to guide future research. Nutr Rev 2016;74:98-117.

4 Birch LL, Marlin DW, Kramer L, et al. Mother-child interaction patterns and the degree of fatness in children. $J$ Nutr Educ 1981;13:17-21.

5 Bruch H. Eating disorders: Obesity, anorexia nervosa, and the person within. Basic Books, 1973.

6 Saltzman JA, Fiese BH, Bost KK, et al. Development of appetite self-regulation: integrating perspectives from attachment and family systems theory. Child Development Perspectives 2018;12:51-7.

7 Anderson SE, Gooze RA, Lemeshow S, et al. Quality of early maternal-child relationship and risk of adolescent obesity. Pediatrics 2012;129:132-40.

8 Wu T, Dixon WE, Dalton WT, et al. Joint effects of child temperament and maternal sensitivity on the development of childhood obesity. Matern Child Health J 2011:15:469-77.

9 Kochanska G, Coy KC, Murray KT. The development of selfregulation in the first four years of life. Child Dev 2001;72:1091-111.

10 Anderson SE, Keim SA. Parent-Child interaction, self-regulation, and obesity prevention in early childhood. Curr Obes Rep 2016:5:192-200.

11 Clark CAC, Woodward LJ, Horwood LJ, et al. Development of emotional and behavioral regulation in children born extremely preterm and very preterm: biological and social influences. Child Dev 2008;79:1444-62.

12 Hughes SO, Power TG, O'Connor TM, et al. Executive functioning, emotion regulation, eating self-regulation, and weight status in low-income preschool children: how do they relate? Appetite 2015;89:1-9.

13 Moding KJ, Augustine ME, Stifter CA. Interactive effects of parenting behavior and regulatory skills in toddlerhood on child weight outcomes. Int J Obes 2019;43:53-61.

14 Bergmeier H, Paxton SJ, Milgrom J, et al. Early mother-child dyadic pathways to childhood obesity risk: a conceptual model. Appetite 2020;144:104459

15 Kong KL, Eiden RD, Paluch RA. Early nonfood parent-infant interactions and development of obesity in a high-risk, diverse sample. Obesity 2019;27:1754-60.

16 Treyvaud K. Parent and family outcomes following very preterm or very low birth weight birth: a review. Semin Fetal Neonatal Med 2014;19:131-5

17 Hack M, Schluchter M, Cartar L, et al. Growth of very low birth weight infants to age 20 years. Pediatrics 2003;112:e30-8.

18 Hales CM, Fryar CD, Carroll MD, et al. Trends in obesity and severe obesity prevalence in US youth and adults by sex and age, 20072008 to 2015-2016. JAMA 2018;319:1723-5.

19 Brown T, Moore THM, Hooper L, et al. Interventions for preventing obesity in children. Cochrane Database Syst Rev 2019;2019.

20 Ogden CL, Fryar CD, Martin CB, et al. Trends in obesity prevalence by race and Hispanic Origin-1999-2000 to 2017-2018. JAMA 2020;324:1208-10.

21 Casey PH, Bradley RH, Whiteside-Mansell L, et al. Evolution of obesity in a low birth weight cohort. J Perinatol 2012;32:91-6.

22 Cunningham SA, Kramer MR, Narayan KMV. Incidence of childhood obesity in the United States. N Engl J Med 2014;370:403-11.

23 Geserick M, Vogel M, Gausche R, et al. Acceleration of BMI in early childhood and risk of sustained obesity. $N$ Engl $J$ Med 2018;379:1303-12.

24 Zheng M, Lamb KE, Grimes C, et al. Rapid weight gain during infancy and subsequent adiposity: a systematic review and metaanalysis of evidence. Obes Rev 2018;19:321-32.

25 Belfort MB, Gillman MW, Buka SL, et al. Preterm infant linear growth and adiposity gain: trade-offs for later weight status and intelligence quotient. J Pediatr 2013;163:e1562:1564-9.

26 Lumeng JC, Taveras EM, Birch L, et al. Prevention of obesity in infancy and early childhood: a national Institutes of health workshop. JAMA Pediatr 2015;169:484-90.

27 Seidler AL, Hunter KE, Johnson BJ, et al. Understanding, comparing and learning from the four epoch early childhood obesity prevention interventions: a multi-methods study. Pediatr Obes 2020;15:e12679.

28 Russell CG, Russell A. A biopsychosocial approach to processes and pathways in the development of overweight and obesity in childhood: insights from developmental theory and research. Obes Rev 2019:20:725-49.

29 Davies WH, Ackerman LK, Davies CM, et al. About your child's eating: factor structure and psychometric properties of a feeding relationship measure. Eat Behav 2007;8:457-63.
30 Putnam SP, Gartstein MA, Rothbart MK. Measurement of finegrained aspects of toddler temperament: the early childhood behavior questionnaire. Infant Behav Dev 2006;29:386-401.

31 MacPhee D. Manual: Knowledge of Infant Development Inventory. Chapel Hill, NC: University of North Carolina, 1981.

32 Radloff LS. The CES-D scale: a self-report depression scale for research in the general population. Appl Psychol Meas 1977;1:385-401.

33 Staples LG, Dear BF, Gandy M, et al. Psychometric properties and clinical utility of brief measures of depression, anxiety, and general distress: the PHQ-2, GAD-2, and K-6. Gen Hosp Psychiatry 2019;56:13-18.

34 Abidin RR. Parenting Stress Index-Short Form. Charlottesville, VA: Pediatric Psychology Press, 1990

35 Bickel G, Nord M, Price C, et al. Guide to measuring household food security, revised 2000. Alexandria, VA: U.S. Department of Agriculture Food and Nutrition Service, 2000.

36 NICHD Early Child Care Research Network. Child care and motherchild interaction in the first three years of life. Developmental Psychology 1999:35:1399-413.

37 Zvara BJ, Mills-Koonce WR, Garrett-Peters P, et al. The mediating role of parenting in the associations between household chaos and children's representations of family dysfunction. Attach Hum Dev 2014;16:633-55.

38 Centers for Disease Control and Prevention (CDC), National Center for Health Statistics (NCHS). National health and nutrition examination survey (NHANES): anthropometry procedures manual. Hyattsville, MD: U.S. department health and human services, centers for disease control and prevention, 2017. Available: https:// wwwn.cdc.gov/nchs/data/nhanes/2017-2018/manuals/2017_ Anthropometry Procedures Manual.pdf

39 Perini TA, GLd O, JdS O, et al. Technical error of measurement in anthropometry. Revista Brasileira de Medicina do Esporte 2005;11:81-5.

40 Berlin KS, Davies WH, Silverman AH, et al. Assessing familybased feeding strategies, strengths, and mealtime structure with the feeding strategies questionnaire. J Pediatr Psychol 2011;36:586-95

41 Ross CE, Mirowsky J. Disorder and decay: the concept and measurement of perceived neighborhood disorder. Urban Affairs Review 1999;34:412-32.

42 Dunsmore JC, Karn MA. Mothers' beliefs about feelings and children's emotional understanding. Early Education and Development 2001:12:117-38.

43 Raes F, Pommier E, Neff KD, et al. Construction and factorial validation of a short form of the Self-Compassion scale. Clin Psychol Psychother 2011;18:250-5.

44 Brownson RC, Hoehner CM, Brennan LK, et al. Reliability of 2 Instruments for Auditing the Environment for Physical Activity. J Phys Act Health 2004;1:191-208.

45 Caldwell BM, Bradley RH. Home Observation for Measurement of the Environment. Little Rock, AR: University of Arkansas at Little Rock, 1984.

46 Matheny AP, Wachs TD, Ludwig JL, et al. Bringing order out of chaos: Psychometric characteristics of the confusion, hubbub, and order scale. J Appl Dev Psychol 1995;16:429-44.

47 Krupsky KL, Parrott A, Andridge R, et al. A mixed methods analysis of environmental and household chaos: implications for childhood obesity prevention through toddlerhood. BMC Public Health. In Press 2021:21:1-9.

48 Raschka C. A Ball for Daisy. New York: Random House, Inc, 2011.

49 Birch LL, Fisher JO, Grimm-Thomas K, et al. Confirmatory factor analysis of the child feeding questionnaire: a measure of parental attitudes, beliefs and practices about child feeding and obesity proneness. Appetite 2001;36:201-10.

50 Jansen E, Williams KE, Mallan KM, et al. The feeding practices and structure questionnaire (FPSQ-28): a parsimonious version validated for longitudinal use from 2 to 5 years. Appetite 2016;100:172-80.

51 Musher-Eizenman D, Holub S. Comprehensive feeding practices questionnaire: validation of a new measure of parental feeding practices. J Pediatr Psychol 2007;32:960-72.

52 Anderson SE, Must A, Curtin C, et al. Meals in our household: reliability and initial validation of a questionnaire to assess child mealtime behaviors and family mealtime environments. J Acad Nutr Diet 2012;112:276-84.

53 Crnic KA, Booth CL. Mothers' and Fathers' Perceptions of Daily Hassles of Parenting across Early Childhood. J Marriage Fam 1991;53:1042-50.

54 Spanier GB. Measuring dyadic adjustment: new scales for assessing the quality of marriage and similar dyads. J Marriage Fam 1976;38:15-28. 
55 Goodman R. Psychometric properties of the strengths and difficulties questionnaire. J Am Acad Child Adolesc Psychiatr 2001;40:1337-45.

56 Gioia GA, Andrwes K, Isquith PK. Behavior Rating Inventory of Executive Function-Preschool Version (BRIEF-P. Psychological Assessment Resources Odessa, FL, 1996.

57 Bradley RH, Caldwell BM. Home observation for measurement of the environment: a revision of the preschool scale. Am J Ment Defic 1979;84:235-44.

58 Dunn DM. Peabody Picture Vocabulary Test. Fifth ed. Pearson, 2018.

59 Mayer M. A Boy, a Dog, a Frog, and a Friend. New York: Penguin Putnam Inc, 1971.

60 Wardle J, Guthrie CA, Sanderson S, et al. Development of the children's eating behaviour questionnaire. J Child Psychol Psychiatry 2001;42:963-70.

61 van Strien T, Frijters JER, Bergers GPA, et al. The Dutch Eating Behavior Questionnaire (DEBQ) for assessment of restrained, emotional, and external eating behavior. Int J Eat Disord 1986;5:295-315.

62 Achenbach TM, Edelbrock C, Burlington V. Child behavior checklist, 1991.

63 Gratz KL, Roemer L. Multidimensional assessment of emotion regulation and dysregulation: development, factor structure, and initial validation of the difficulties in emotion regulation scale. $J$ Psychopath Behav Assess 2004;26:41-54.
64 Bernstein DP, Fink L, Handelsman L, et al. Initial reliability and validity of a new retrospective measure of child abuse and neglect. Am J Psychiatry 1994;151:1132-6.

65 NutritionQuest. Block Questionnaire for Ages 2-7 - Kids 2-7 FFQ. Available: https://www.nutritionquest.com/assessment/list-ofquestionnaires-and-screeners/ [Accessed 12 Oct 2021].

66 Weintraub S, Dikmen SS, Heaton RK, et al. Cognition assessment using the NIH Toolbox. Neurology 2013;80:S54-64.

67 WHO Multicentre Growth Reference Study Group. WHO child growth standards: Length/height-for-age, weight-for-age, weight-for-length, weight-for-height and body mass index-for-age: methods and development. Geneva: World Health Organization, 2006

68 Sullivan JA, Zvara BJ, Keim SA, et al. Knowledge of infant development and parent well-being: cross-sectional analysis of toddlers. J Dev Behav Pediatr 2021;42:442-9.

69 Khalsa A, Weber Z, Keim S. Factors associated with parenting stress in parents of 18 month old children. Child: Care Health Dev. In Press 2021.

70 Sipola-Leppänen $M$, Vääräsmäki $M$, Tikanmäki $M$, et al. Cardiometabolic risk factors in young adults who were born preterm. Am J Epidemiol 2015;181:861-73.

71 Census US. Quick facts Columbus Ohio; United States, 2018. Available: https://www.census.gov/quickfacts/fact/table/ columbuscityohio,US/PST045218 [Accessed 11 Feb 2020]. 